\title{
Estudo avaliativo da abordagem numérica do traçamento de raios na modelagem sísmica de tempos de trânsito
}

\author{
Rodrigo de Santana Santos, ANP/UFBA/CPGG \\ Wilson M. Figueiró, CPGG-IGEO-UFBA, Salvador, BA, Brasil
}

Copyright 2014, SBGf - Sociedade Brasileira de Geofísica.

Este texto foi preparado para a apresentação no VI Simpósio Brasileiro de Geofísica, Porto Alegre, 14 a 16 de outubro de 2014. Seu conteúdo foi revisado pelo Comitê Técnico do VI SimBGf, mas não necessariamente representa a opinião da SBGf ou de seus associados. É proibida a reprodução total ou parcial deste material para propósitos comerciais sem prévia autorização da SBGt.

\begin{abstract}
Seismic waves traveltime modeling is performed by ray tracing. In this work, it is studied a numerical approach of seismic wave ray tracing on models of isotropic compressional wave velocity fields, that simulate geological situations. The ray tracing is done by means of ray equations solution (Červeny, 2005), in which generally, is found by approximative numerical methods. As such methods introduce erros to the adapted procedure, the aim here is to evaluate the accuracy of times by such methods.
\end{abstract}

\section{Resumo}

A modelagem dos tempos de trânsito das ondas sísmicas é realizada atravéz de traçamento de raios. Neste trabalho avalia-se a abordagem numérica do traçamento de raios das ondas sísmicas em modelos de campos de velocidades de ondas compressionais e isotrópicas, que simulam situações geológicas. O traçamento de raios é feito com base na solução das equações do raio (Červený, 2005), que na maioria das situações é encontrada por métodos numérico aproximativos. Como métodos de aproximação introduzem erros ao procedimento, a proposta aqui estabelecida é avaliar a acurácia de tempos gerados por meio desses métodos.

\section{Introdução}

Modelagens sismológicas que usam traçamento de raios sísmicos são usadas para construção de imagens da subsuperfície que são ferramentas amplamente utilizadas na indústria de petróleo e em laboratórios de pesquisa deste ramo na exploração de hidrocarbonetos. A modelagem fornece uma noção de como cada parâmetro físico influência nos dados adquiridos em campo, de forma que se pode explicar observações de tempos de trânsito por meio de resultados de modelagens. Ao longo do trajeto dos raios sísmicos é possível calcular os tempos de trânsito das ondas compressionais, e assim esses tempos são usados em trabalhos de inversão sísmica, sendo estes de grande préstimo na tomografia sísmica.

A modelagem é feita em campos de velocidades sísmica parametrizados por funções matemáticas, de tal forma que quando se aplica tais funções nas equações do raio, o resultado é um conjunto de
EDO's (equações diferenciais ordinárias), que quando devidamente resolvidas geram os traçados dos raios e os tempos de trânsito. $\mathrm{Na}$ maior parte das situações, a solução dessas equações é obtida numericamente, e portanto surgem questionamentos quanto a acurácia de tal abordagem. Portanto, faz-se necessário avaliar tal aproximação, buscando fornecer resposta para a pergunta: qual é o grau de aproximação obtido por métodos numéricos de traçamento de raios sísmicos normalmente utilizados em trabalhos de modelagem sísmica?

Neste trabalho o método numérico adotado foi o de Euler que é amplamente utilizado em trabalhos deste ramo. A resposta ao questionamento levantado é dada por meio da interpretação de resutados obtidos com dois procedimentos diferentes. No primeiro caso é feito uma comparação entre resultados obtidos com a resolução analítica das equações do raio, com os resultados encontrados por meio do método aproximativo. A segunda maneira de avaliação é feita por meio da verificação da equação eikonal que deve ser satisfeita ao longo do raio em todos os pontos do modelo do campo de velocidades.

\section{Equação Eikonal e Equações do Raio}

Muitas abordagens diferentes podem ser usadas para definir a obtenção de sistemas de traçamento de raios. A abordagem mais geral e, consequentemente, a que será usada aqui, é baseada na solução de alta frequência assintótica da equação da onda elastodinâmica, que resulta na equação eikonal, que em coordenadas cartesianas assume a seguinte forma:

$$
\rho_{i} \rho_{i}=\frac{1}{V\left(x_{i}\right)^{2}} \quad \text { onde } \quad \rho_{i}=\frac{\partial T}{\partial x_{i}} \quad i=1,2 \text { e } 3,
$$

onde $T=T\left(x_{i}\right)$ é o tempo de trânsito, $\rho_{i}$ são os componentes do vetor vagarosidade $\vec{\rho}=\nabla T$ na posição $\vec{\chi}=\left(x_{1}, x_{2}, x_{3}\right)$ e $V\left(x_{i}\right)$ é a velocidade da onda primária $v=\alpha$. A Eq. (1) pode ser apresentada pelo hamiltoniano como $\mathscr{H}\left(x_{i}, \rho_{i}\right)=0$, onde $\mathscr{H}$ pode assumir varias formas diferente como $\mathscr{H}\left(x_{i}, \rho_{i}\right)=\left(\rho_{i} \rho_{i}-V^{-2}\right)$, ou $\mathscr{H}\left(x_{i}, \rho_{i}\right)=\left(\rho_{i} \rho_{i}^{1 / 2}-V^{-1}\right)$ ou ainda $\mathscr{H}\left(x_{i}, \rho_{i}\right)=\frac{1}{2}\left(V^{2} \cdot \rho_{i} \rho_{i}-1\right)$. O sistema de equações do raio é encontrado pela substituição de umas das formas do hamiltoniano no sistema característico (Bleitein, 1984), e esse sistema é resolvido em termos de uma característica. No trabalho em questão essa característica é a trajetória $x_{i}=x_{i}(u)$ no espaço 3D, ao longo do qual $\mathscr{H}\left(x_{i}, \rho_{i}\right)=0$ é satisfeito, onde $u$ é o parâmetro da trajetória. A equação do hamiltoniano que será adotada neste trabalho é $\mathscr{H}=1 / n \cdot\left(\rho_{i} \rho_{i}^{n / 2}-V\left(x_{i}\right)^{-n}\right)$, onde o valor de $n$ determina o parâmetro ao longo do raio. 
Após substituir essa equação no sistema característico e fazer $n=2$ o resultado é o sistema de equações do raio dada por Eq. (2). Para $n=2$ se tem que $u=\tau$, onde $\tau$ é chamado de variável natural ao longo do raio, o parâmetro $\tau$ não possui significado físico, e tem como dimensão no sistema internacional de medidas $\mathrm{m}^{2} / \mathrm{s}$.

$$
\frac{d x_{i}}{d \tau}=\rho_{i}, \quad \frac{d \rho_{i}}{d \tau}=\frac{1}{2} \frac{\partial}{\partial x_{i}}\left(\frac{1}{V^{2}}\right), \quad \frac{d T}{d \tau}=\frac{1}{V^{2}} \quad i=1,2 \text { e } 3 .
$$

\section{Traçamento dos Raios e Cálculo de Tempos}

Após substituir a equação do modelo campo de velocidades na Eq. (2) é gerado um conjunto de equações do raio para o modelo referido. Quando as equações do raio são resolvidas resultam em funções que fornecem a posição do raio e o tempo que o mesmo gastou para percorrer da fonte até tal posição. Assim, podem ser encontrados todos os pontos do caminho que o raio percorre de um ponto a outro do modelo. O método de resolução das equações do raio pode ser analítico, quando encontra-se uma solução para tais equações algebricamente, ou pode ser numérico, quando a solução é obtida numéricamente e tem caráter aproximado. Neste trabalho o traçado dos raios é feito de ambas as formas descritas acima. O método numérico usado no traçamento aproximado aqui foi o método de Euler, que é o correspondente ao método de Taylor truncado após a primeira derivada, que também pode ser visto como o método de Runge-Kutta de primeira ordem (Butcher, 1987). A escolha deste método se deu pelo fato do mesmo ser grandemente usado em atividades de pesquisa, que usam traçamento de raio, como em Figueiró et al., 2005; Mendes, 2009; de Souza \& Figueiró, 2004; Santos, 2008; Santos, 2006; e Teles, 2004. Tal método aplicado nas equações do raio encontradas para $n=2$, é feito partindose de uma posição conhecida $\vec{\chi}\left(\tau_{0}\right)$ com uma direção também conhecida $\vec{\rho}\left(\tau_{0}\right)$, e daí então, cada nova posição e direção é encontrada, como visto em Eq. (3).

$$
\left\{\begin{array}{l}
\vec{\chi}\left(\tau_{0}+\Delta \tau\right) \approx \vec{\chi}\left(\tau_{0}\right)+\vec{\rho}\left(\tau_{0}\right) \cdot \Delta \tau \\
\vec{\rho}\left(\tau_{0}+\Delta \tau\right) \approx \vec{\rho}\left(\tau_{0}\right)+\frac{1}{2} \nabla\left(\frac{1}{V^{2}\left(\vec{\chi}\left(\tau_{0}\right)\right)}\right) \cdot \Delta \tau
\end{array}\right.
$$

onde $\Delta \tau$ é o passo. O raio é uma linha poligonal, onde os nós são as posições $\vec{\chi}\left(\tau_{0}+K . \Delta \tau\right)$, onde $K$ é um inteiro positivo, que representa o número de passos desde a fonte até o registro. O tempo gasto entre cada nó é calculado dividindo-se a distância entre esses nós pela velocidade média, e a soma de todos os tempos entre os nós da poligonal resulta no tempo numérico total gasto pelo raio. Para a solução analítica pode-se usar algum método conhecido na literatura (Marcia, 2010; Harirer, 1971), o que resulta nas equações paramétricas da posição do raio e também e na equação dos tempos de trânsito para o raio chegar a tal posição. Esta solução é feita resolvendo as equações do raio, e é complementada com as condições iniciais (posição e direção de saída do raio), de tal forma que para cada condição dada determina-se um único raio. Afim de tornar os resultados mais didáticos as variáveis $x_{1}, x_{2}, x_{3}, p_{1}, p_{2}$ e $p_{3}$ serão daqui para frente trocadas, respectivamente, por $x, y, z, p_{x}, p_{y}$, e $p_{z}$. Neste trabalho os raios são coplanares, sendo que estes estão limitados ao plano $y=0$ com $p_{y}=$ cte, deste modo a trajetórtia do raio assim como suas equações se reduzem ao plano 2-D.

\section{Avaliação da Acurácia do Método Numérico}

É sabido que um processo numérico acumula erros durante seu proceder, e avaliar quantitativamente esses erros é uma maneira de avaliação da acurácia deste procedimento. Para tal avaliação foram feitos em cada experimento três testes diferentes, o que resultou em dados a serem interpretados. As primeira e segunda maneiras de realização dos testes, leva em conta o traçado dos raios e tempos de trânsito feitos analiticamente, pois essa abordagem apresenta dados calculados exatos, de tal forma que podem ser usados como base para a análise dos dados numéricos. Como se vê na Fig 1, os raios numéricos e analíticos são registrados em posições diferentes, e assim possuem tempos de trânsito diferentes. Sabendo-se disto traça-se gráficos de desvio relativo entre os tempos executados pela abordagem numérica e pela analítica calculados pela Eq. 4, e também gráficos das distâncias entre as posições de chegadas, em função do ângulo de saída calculado pela Eq. 5 .

$$
D_{t}(\theta)=\left|\frac{T_{A}-T_{N}}{T_{A}}\right| \cdot 100 \%,
$$

onde $T_{A}$ são os tempos calculados de maneira analítica, $T_{N}$ são os tempos numérico, |.| é o modulo e $D_{t}(\theta)$ representa o desvio relativo entre esses tempos de trânsito.

$$
D_{r}(\theta)=\left\|\vec{\chi}_{A}-\vec{\chi}_{N}\right\|
$$

com $\vec{\chi}_{A}$ sendo a posição que o raio analítico é recebido, e $\vec{\chi}_{N}$ o local de medição do raio numérico numérico, \|. . é a função norma e $D_{r}(\theta)$ a distância em quilômetros. A terceira forma de se avaliar a acurácia do traçado numérico é por meio da equação eikonal $\mathscr{H}\left(x_{i}\right)=0$, que deve ser satisfeita ao longo do raio. Como a atualização do vetor vagarosidade é numérica, existirá um erro calculado pela Eq. 6, que é associado a cada posição do modelo visitada pelo raio. Com esses erros pode-se criar campos de erros com relação ao vetor vagarosidade, para cada modelo estudado.

$$
\delta(x, z)=\left|\rho_{i} \rho_{i}-V^{-2}\right|,
$$

com $\delta(x, z)$ sendo os erros referentes a equação eikonal, que são os erros no vetor vagarosidade.

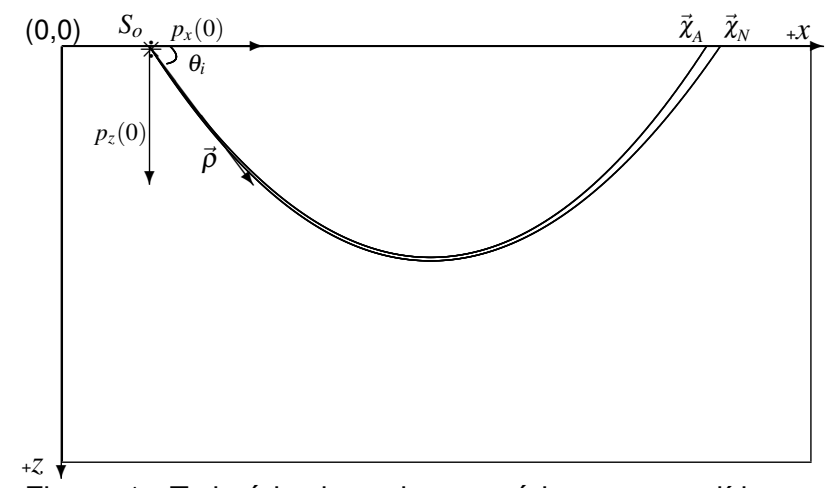

Figura 1: Trajetória dos raios numérico $r_{N}$ e analítico $r_{A}$, que partem da fonte $S_{0}$ com mesmo ângulo de saída $\theta$. 


\section{Resultados}

Para realização dos experimentos foram selecionados dois modelos bidimensionais e isotrópico de campos velocidades de ondas compressionais, que são parametrizados por funções das variáveis $x$ e $z$. Nesses campos, as velocidades são dadas em quilômetro por segundo $(\mathrm{km} / \mathrm{s})$. O primeiro modelo, nomeado por $M_{1}$, representa um meio de camadas plano paralelas inclinadas, e pode ser visto na Fig. 2, e este é parametrizado pela Eq. (7). Já no segundo caso, o modelo é o $M_{2}$ e representa um meio homogêneo que recebeu uma intrusão de um corpo anômalo, como pode ser visto na Fig. 3, e é representado pela Eq. (8).

$$
\begin{gathered}
V(x, z)=\frac{1}{\sqrt{A_{1}+B_{1} \cdot x+C_{1} \cdot z}} . \\
V(x, z)=\frac{1}{\sqrt{A_{2}+B_{2} \cdot x^{2}+C_{2} \cdot z^{2}+F_{2} \cdot x+G_{2} \cdot z}},
\end{gathered}
$$

onde $A_{1}=0,2 ; B_{1}=-0,01803 ; C_{1}=-0,07063 ; A_{2}=$ 0,$753 ; B_{2}=0,0983 ; C_{2}=0,764 ; F_{2}=-0,786$ e $G_{2}=-0,7869$.

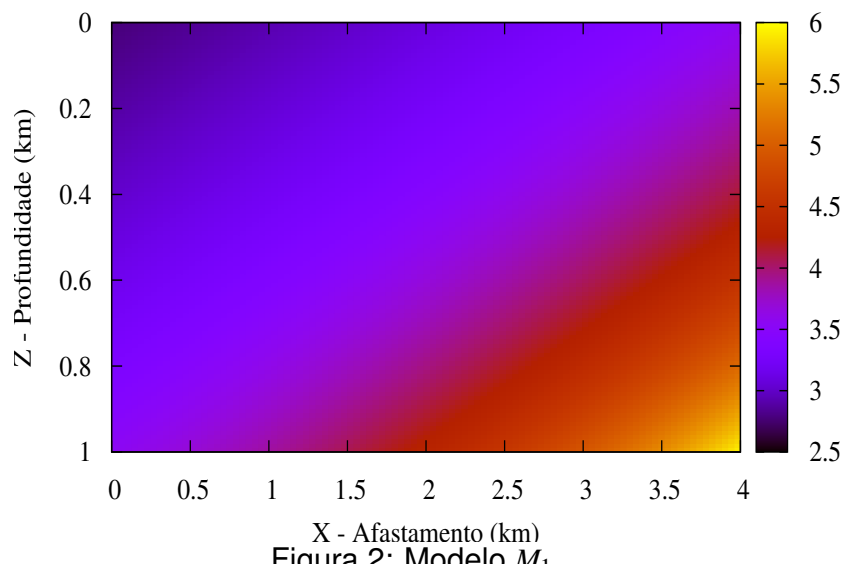

Figura 2: Modelo $M_{1}$.

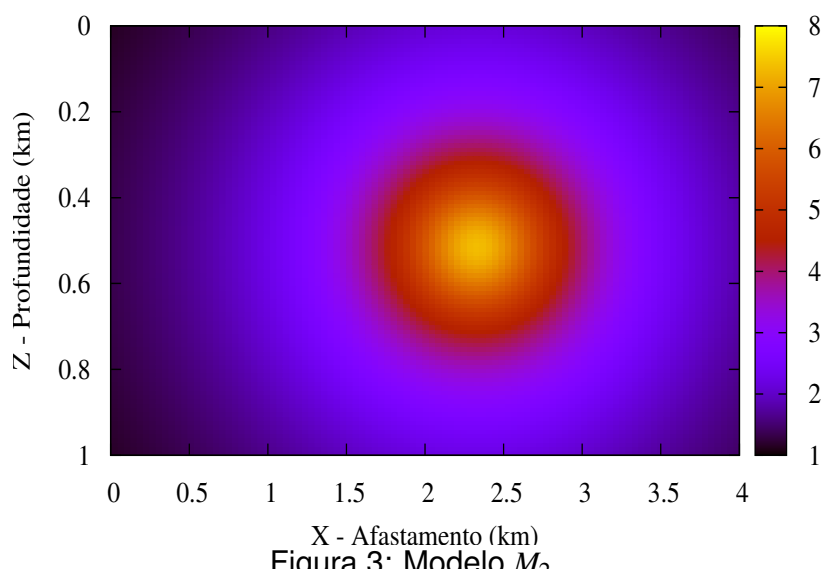

O traçamento foi feito detonando uma fonte em uma determinada posição na superfície $\vec{\chi}=\left(S_{0}, 0\right)$, e coletando dados calculados de noventa raios que partem da fonte com diferentes condições iniciais. Para o modelo $M_{1}$ $S_{0}=1,0$ e para o modelo $M_{2}, S_{0}=2,2$. As Figs. 4 e 5 mostram os campos de raios para os traçamentos feitos com abordagem numérica e analítica, respectivamente, para o modelo $M_{1}$, e as Figs. 6 e 7 mostram esses traçados quando feitos no modelo $M_{2}$.

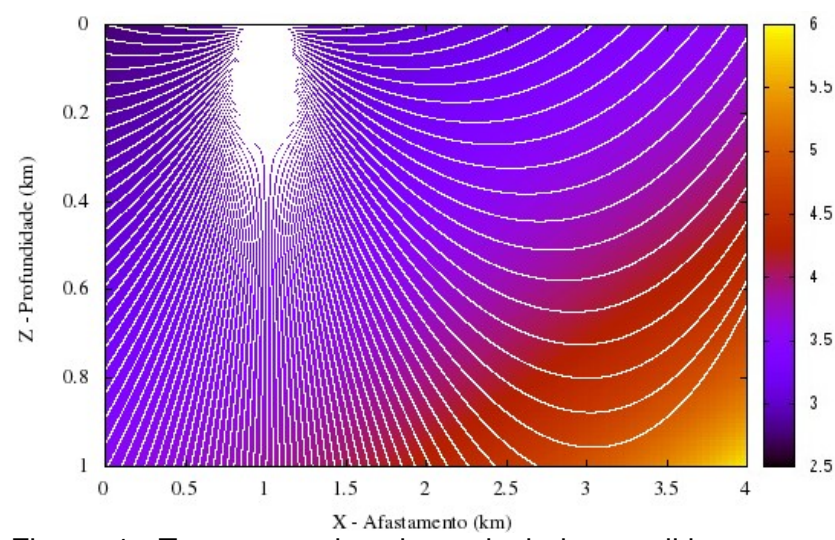

Figura 4: Taçamento de raios calculados analiticamente para fonte colocada na posição $S_{0}=1,0$ do modelo $M_{1}$.

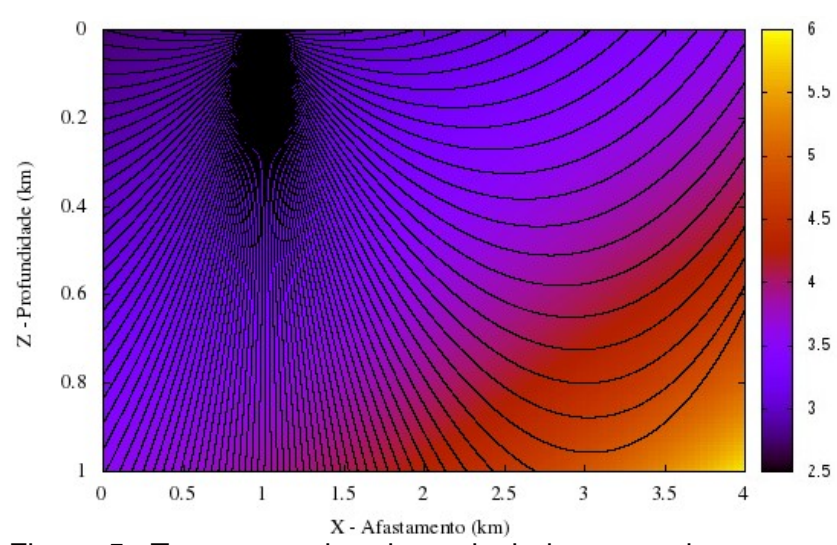

Figura 5: Taçamento de raios calculados numericamente para fonte colocada na posição $S_{0}=1,0$ do modelo $M_{1}$.

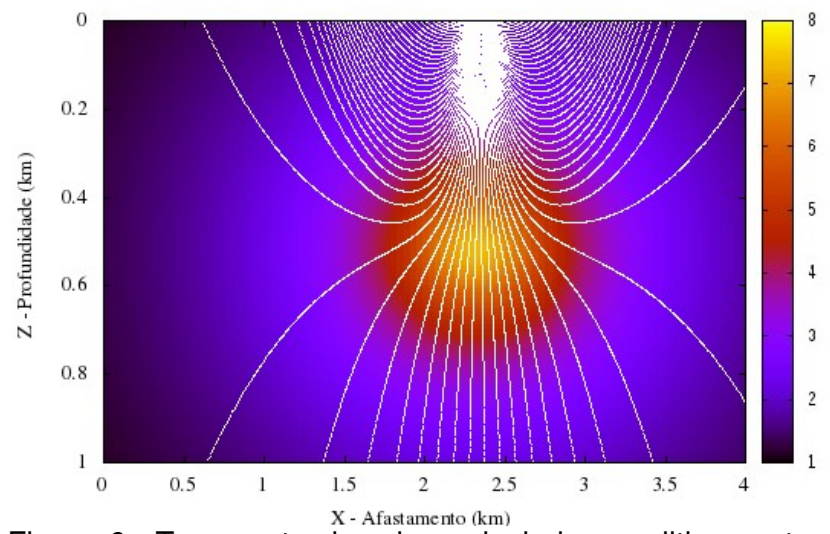

Figura 6: Taçamento de raios calculados analiticamente para fonte colocada na posição $S_{0}=2,5$ do modelo $M_{2}$.

Como dito, as posições de chegada dos raios numéricos e analíticos são diferentes. Para os modelos $M_{1} \mathrm{e}$ $M_{2}$, respectivamente, são mostradas as Figs. 8 e 9 que exibem as distâncias entre as posições de registro 
dos raios numéricos e analíticos, que partem com as mesmas condições iniciais. Nestes gráficos cada raio é individualizado por meio do ângulo de saída da fonte, e estes ângulos são dados em graus.

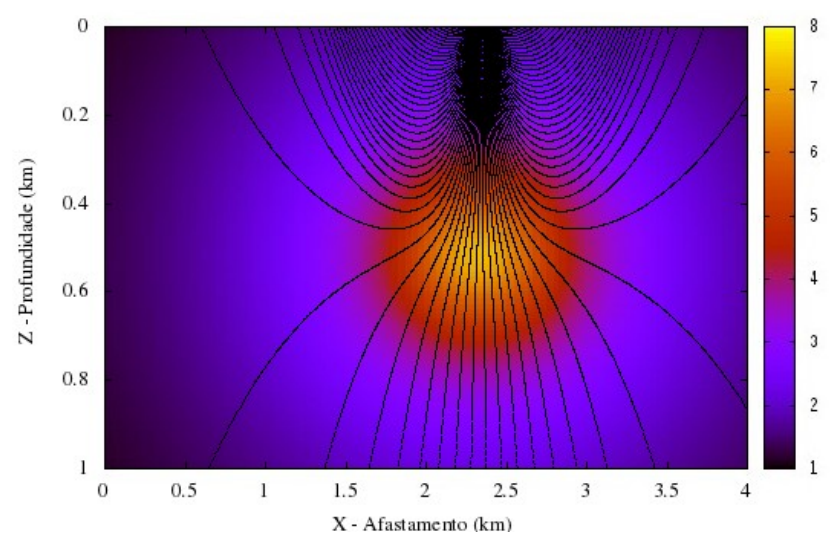

Figura 7: Traçamento de raios calculados numericamente para fonte colocada na posição $S_{0}=2,5$ do modelo $M_{2}$.

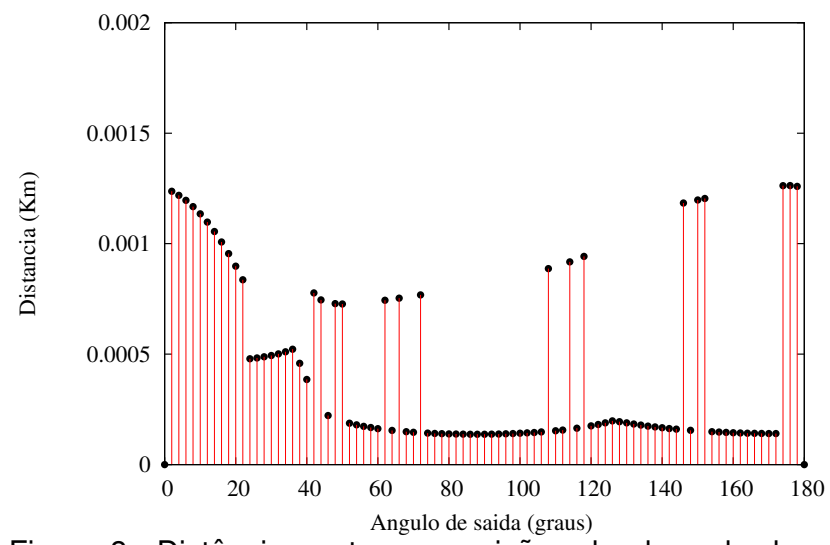

Figura 8: Distâncias entre as posições de chegada dos raios numérico e analtico originados com as mesmas condições iniciais no modelo $M_{1}$.

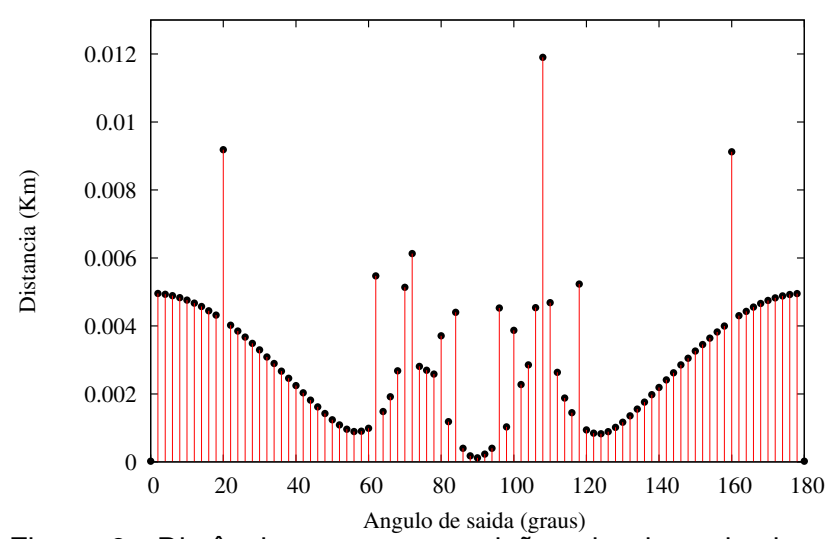

Figura 9: Distâncias entre as posições de chegada dos raios numérico e analtico originados com as mesmas condições iniciais no modelo $M_{2}$.

As Figs. 10 e 11 mostram, respectivamente, os tempos de trânsito dos raios numéricos e analíticos referentes ao modelo $M_{1}$ vistos nas Figs. 4 e 5 . Analogamente as Figs. 10 e 11 mostram os tempos de trânsito dos campos de raios mostrados nas Figs. 4 e 5, que são referentes ao modelo $M_{2}$.

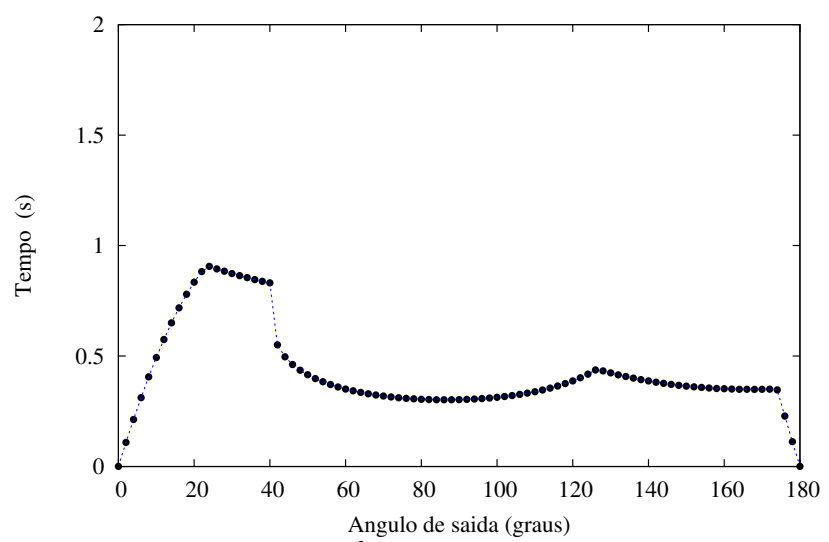

Figura 10: Tempos de trânsito calculados analiticamente para fonte colocada na posição $S_{0}=1,0$ do modelo $M_{1}$.

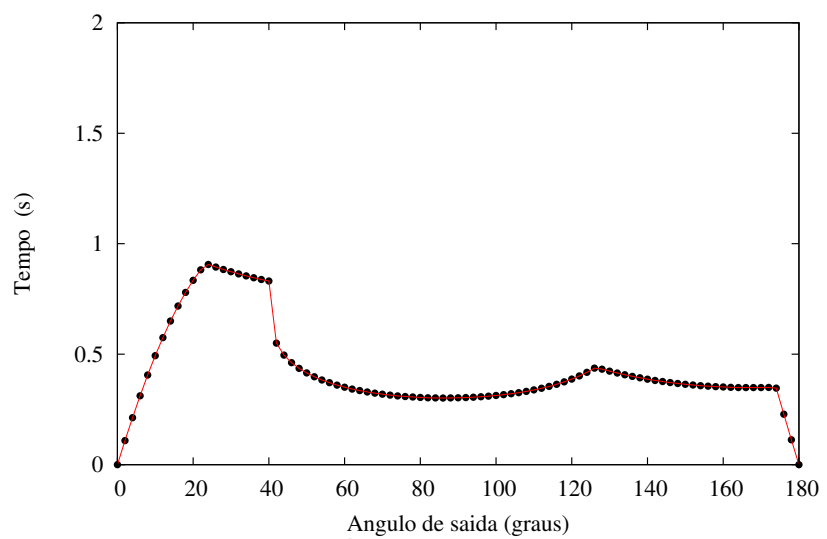

Figura 11: Tempos de trânsito calculados numericamente para fonte colocada na posição $S_{0}=1,0$ do modelo $M_{1}$.

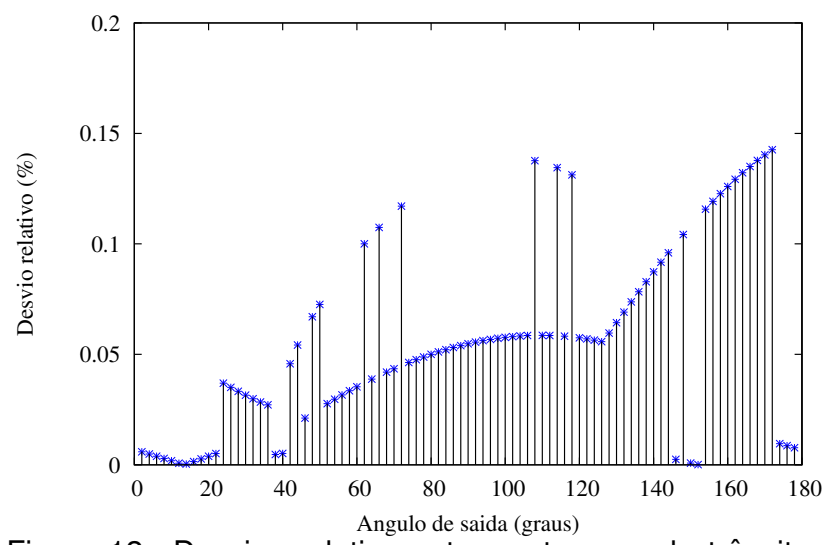

Figura 12: Desvios relativo entre os tempos de trânsito calculados analiticamente e numericamente, para raios originados com mesmas condições iniciais no modelo $M_{1}$.

Os campos de erros do vetor vagarosidade mostrados nas Figs. 16 e 17 para os modelos $M_{1}$ e $M_{2}$, respectivamente, foram gerados colocando-se a fonte em quarenta posições diferentes e fazendo-se partir noventa raios com distância angular constante entre si, e usando resultados da Eq. 6 em cada posição visitada pelo raio. Fazendo-se assim pôde-se cobrir o máximo possível de cada campo de 
velocidades e gerar campos de erros com maiores graus de credibilidade.

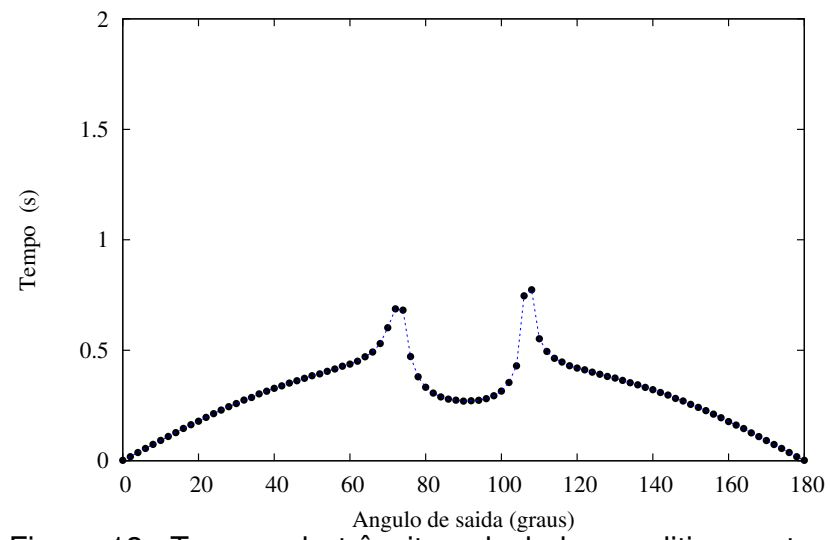

Figura 13: Tempos de trânsito calculados analiticamente para fonte colocada na posição $S_{0}=2,5$ do modelo $M_{2}$.

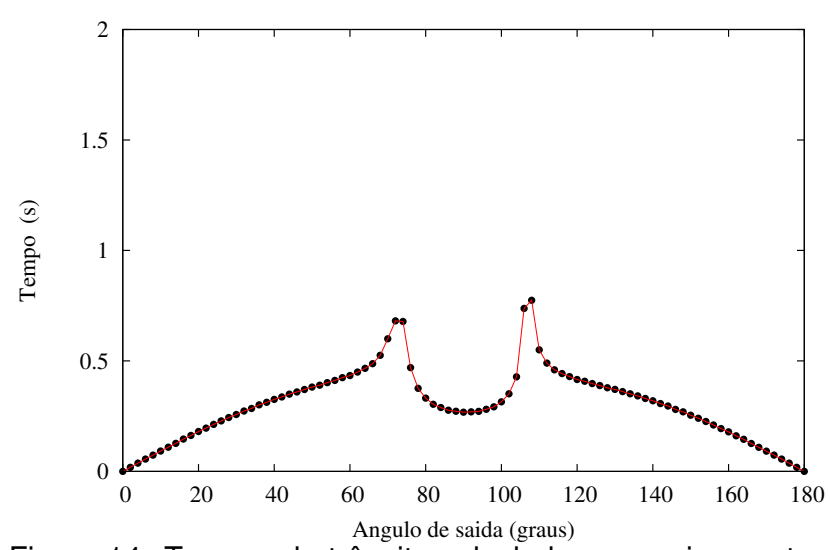

Figura 14: Tempos de trânsito calculados numericamente para fonte colocada na posição $S_{0}=2,5$ do modelo $M_{2}$.

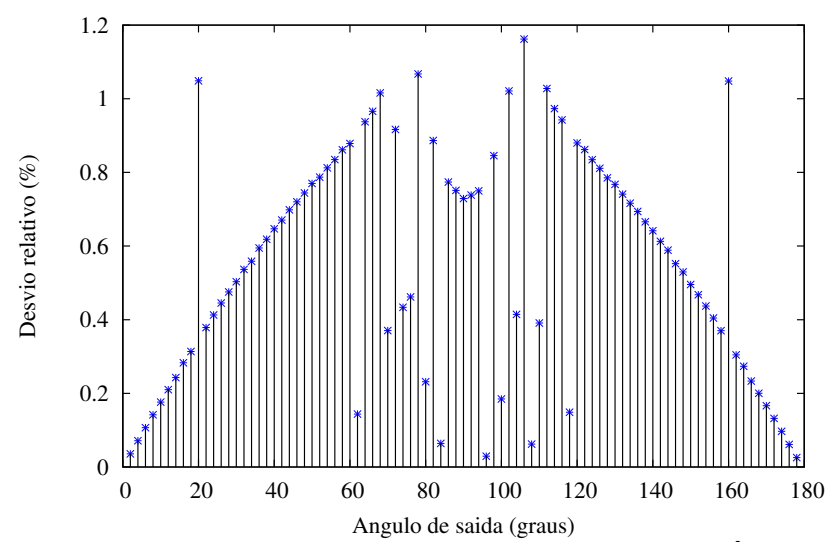

Figura 15: Desvios relativo entre os tempos de trânsito calculados analiticamente e numericamente, para raios originados com as mesmas condições iniciais no modelo $\mathrm{M}_{2}$.

As Figs. 12 e 15 mostram os desvios relativos entre os tempos de trânsito numérico e analíco, para os modelos $M_{1}$ e $M_{2}$, respectivamente.

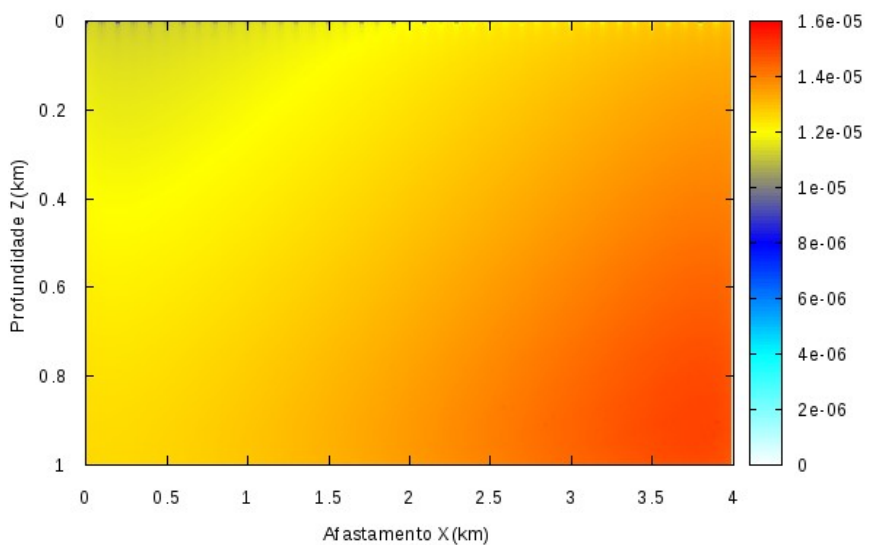

Figura 16: Campo de erros do vetor vagarosidade, relacionado ao Modelo $M_{1}$

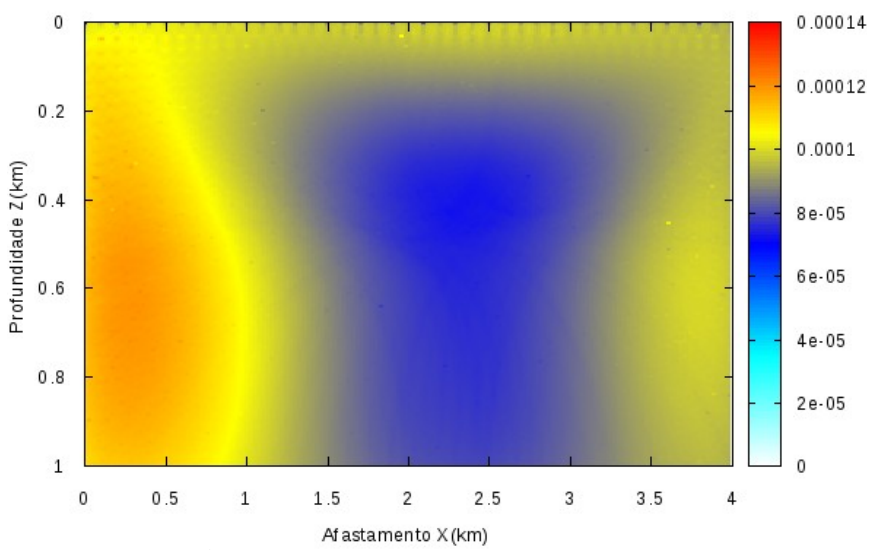

Figura 17: Campo de erros do vetor vagarosidade, relacionado ao Modelo $M_{2}$.

\section{Discussão e Conclusões}

O modelo da intrusão granítica é um campo de velocidades que possui função matemática que produz variações bruscas de velocidades nas vizinhanças do corpo, assim os raios quando se aproximam desta região sofrem mudança brusca na direção de propagação, de tal forma que poucos raios conseguem atravessar tal corpo, e este fator faz com que sejam coletados dados de poucos pontos que se situam abaixo dele, e assim o programa usado para desenhar o campo de erros deste modelo, usa os pontos que possui e faz interpolação nas regiões sem registro. É por esta razão que ao analisar a Fig 17, pode-se perceber que os erros abaixo deste corpo são semelhante aos erros logo acima, isto pois são os locais mais bem visitados pelos raios.

O modelo das camadas plano paralelas inclinadas, por sua vez é parametrizado por uma função matemática que possui um grau de complexidade muito menor que o modelo da intrusão granítica, e assim quando se analisa o campo de erros do modelo $M_{1}$, pode-se notar que os erros anexados ao vetor vagarosidade pelo procedimento numérico são bem menores que para o modelo $M_{2}$. No caso do campo de erros gerado pelo modelo $\mathrm{M} 2$, os maiores erros estão ligados as menores velocidades, já no caso do modelo M1, o campo de erros do modelo apresenta um certo grau de homogeneidade, e ocorre que os erros ligeiramente maiores estão nas regiões de 
maiores velocidades, é o inverso do que ocorre com o campo de erros do modelo $M_{2}$.

Quando se analisa os erros nos tempos e nas posições de chegada dos raios, para ambos os modelos $M_{1}$ e $M_{2}$ nota-se que a complexidade do meio é função determinante na acurácia do método aproximativo, pois comparação das figuras dos erros nos tempos de trânsito como também nas figuras dos erros nas posições, para os modelos $M_{1}$ e $M_{2}$, mostra que os dados do modelo $M_{1}$ são sempre mais acurados que os do modelo $M_{2}$, e isto nos leva a concluir que a acurácia apresentada pelo método será função da complexidade do modelo usado.

Como esperado em ambas as situações os procedimentos aproximativos exibem erros, porém mesmo usando um campo de velocidades com um grau de complexidade elevado como $M_{2}$, pode-se dizer que os dados calculados são de boa qualidade, isto pois todos os gráficos usados nas análises dos erros não apresentaram valores tão elevados quando comparado as dimensões dos modelos usados. Assim, com os resultados obtidos e com a metodologia empregada pode-se concluir qualificando positivamente o método numérico de Euler como um método satisfatório para o traçamento de raios na modelagem sísmica de tempos de trânsito, ou seja, tal metodologia pode ser usada sem grande perdas nas aproximações.

\section{Agradecimentos}

Os autores agradecem ao CPGG/UFBA/ANP, pelo apoio no desenvolvimento deste trabalho. Rodrigo de Santana Santos agradece o ANP pelo suporte financeiro através de bolsa de pesquisa.

\section{Referências}

Červeny, V.; 2005. Seismic ray theory. Cambridge University Press.

Bleitein, N.; 1984. Mathematical methods for wave phenomena. Academic press.

Butcher, J.C.; 1987. The numerical analysis of ordinary differrential equations: Runge-Kutta and general linear methods, Wiley-Interscience.

Figueiró, W.M.; Oliveira, S.P. e Novaes, F.C. (2005) Traveltime data profiles obtained using seismic ray tracing methods for the continetal slope model parameterized by polynomials, In: 9th International Congress of the Brazilian Geophysial society, Expanded Abstract, Salvador, BA, Brazil.

Márcia S.; Marica C.M. 2010. Tópicos : Séries e equações Diferenciais. EDUFBA, Salvador, Brasil.

Mendes, F.C.; 2009. Modelagem sísmica de curvas de tempos de trânsito usando traçamento de raios em campos de velocidades parametrizados por polinômios. Trabalho de graduação em Geofísica, UFBA.

Hairer, E.; Norsett, S.P. e Wanner, G.; 1991. Solving ordinary differential equations, Vol 2. Springer.

Santos, M.A.B.; 2008. Modelagem sismologica de curvas de tempos de trânsito com parametrização por série trigonometrica do campo global de velocidades. Trabalho de graduação em Geofísica, UFBA.
Santos, R.H.M. e Figueiró, W.M.; 2006. Modelagem acústica bidimensional usando diferentes parametrizações de campos de velocidades, Revista Brasileira de Geofísica. 24 (1): 103-105.

de Souza A.E.C.M. e Figueiró, W.M.; 2004. Campos de tempos de trânsito obtidos por traçamento de raios sísmicos em campos de velocidades com parametrização polinomal, In: I Simpósiso Brasileiro de Geofísica, Resumos Expandidos, São Paulo, SP, Brasil.

Teles, M.R.R. e Figueiró, W.M.;2009. Modelagem sísmica de tempos de trânsito de reflexão em interface parabólica, In: 11th International Congress of the Brazilian Geophysial society, Expanded Abstract, Salvador, BA, Brazil. 